\title{
Intelligent and Interactive Companion Combined With Wearable Technology and Re-creatable Environment to Avoid Anxiety
}

\author{
Hashini Senaratne \\ University of Moratuwa \\ Moratuwa, 10400, Sri Lanka \\ hashini.10@cse.mrt.ac.lk
}

\begin{abstract}
With the modernization of the world, people have led into more stressful lifestyles. Due to long lasting stress, many individuals experience anxiety conditions and this problem has become a common problem in every society of the world. To solve this issue, technology has come into play with suggestions of different types of solutions. In this paper we have come up with a proposal that can become a future work in the human computer interaction field, which suggests an intelligent and interactive companion supported with wearable technology to monitor the stress level of the user and a controllable environment with the potential of recreating moments, in order to avoid anxiety. Although there are existing wearable devices that help to monitor stress levels and some initial level research has been done to come up with companions to reduce stress, integration of a wearable device along with an interactive companion will be a newer level of research. Also identifying and capturing environmental factors which affects one's stress level and changing environmental factors to reduce the stress level, is a less studied area. Therefore the suggested future work exposes a research area which has the capability of expanding a lot in the future.
\end{abstract}

Anxiety; Companion; Health; Re-creatable Environment; Wearable Technology; Wellbeing.

\section{A PROBLEM THAT RUNS ACROSS EVERY NATION}

Anxiety has become one of the most common mental problems in the modern world, which also causes other types of mental disorders such as depression. In 2014, $19.7 \%$ of the people in United Kingdom aged 16 and older have showed symptoms of anxiety or depression, which is a $1.5 \%$ increase from 2013 [1]. It has been found that in Australia from 2014 to 2015 , one in eight females (13.0\%) and one in ten males $(9.4 \%)$ reported having an anxietyrelated condition [2]. Anxiety is one of the negative effect of stress, which makes a person feel fear and uneasiness along with side effects such as chest pains, shortness of breath, stomachaches and dizziness. Long term stress and anxiety directly affect a person's physical and mental health negatively and can lead to diseases like heart diseases, high blood pressure, migraine and sleeping disorders.

Ultimately, anxiety reduces the life expectancy of an individual and can be life threatening as it can cause suicides. According to the World Health Organization's first global report on suicide prevention which was released in 2014, the countries Guyana, Democratic People's Republic of Korea and Republic of Korea ranked at the top with respective suicide rates of 44.2, 38.5 and 28.9 where the measure is given per 100,000 [3]. It has been discovered that among individuals reporting a lifetime history of suicide attempt, over $70 \%$ had an anxiety disorder [4]. Hence it can be concluded that anxiety is one of the major causes behind the increased number of suicides. Also a study in 2012 has found that globally 1 in 13 suffers from anxiety [5]. Therefore anxiety has become a problem that grows across almost every nation.

\section{RELATED WORK}

The longer the stress lasts, more difficult it is to overcome the problems ahead. Since this has become a crucial issue nowadays, there are many mobile apps released to the market in order to promote mindfulness and meditation. Apart from the plenty of applications available in mobile market which attempt to make people's lives healthier by making them follow guided plans and exercises, some researchers explore the use of an interactively guided loving-kindness meditation focused on providing external visualizations [6]. Taking another 
step ahead, wearable technology has come into the play in order to monitor the stress level of a person. Projects like Spire [7] and Embrace [8] monitor the stress level of an individual by measuring different attributes such as respiration patterns, heart rate and skin conductance and also suggest the activities they can perform when stressed moments are monitored. WeaRelaxAble is a design proposed to provide various feedback modalities, such as vibration, ambient light, acoustic stimuli and heat in order to reduce the user's stress level, which includes with a shirt to wear by the user and a wrist worn device to trigger the stimuli [9].

Some initial research has been carried out to reduce the stress level using robotic companions. Emobie is an ongoing project with the intention of producing a social robot that provides companionship and comfort to children with anxiety [10]. Paro is a seal robot which is produced to reduce the stress level and improve psychological outcomes for elderly residents in a care-house [11]. Huggable is a social robot designed to mitigate stress, anxiety and pain in children and parents at pediatric hospitals [12]. These studies show that a pet like companion can become a stress and anxiety reducer for the user.

Although various kinds of work are being carried out in this area, still there are many ways to extend helpful hands to the society with the use of technology to reduce growing stress levels. The existing solutions address only few concerns, as well as the audience considered is narrow. Most of the solutions are aiming literate audiences and not interactive enough. Still there are open opportunities to improve the accuracy in detecting the stress level variations, through studies. Therefore it is clear that there is a high demand for interactive, user friendly, easily accessible and accurate solutions which helps to maintain good health with a reduced stress level. Proposed idea is to come up with a solution which extends further opportunities to deal with stress in a more practical way.

\section{THE ENVISIONED SYSTEM}

The proposed project mainly aims a usual audience varies from teenagers to adults. It also aim to customize the system especially for children. The suggested framework consists of three main components as follows.

(i) Wearable device which collects body data

(ii) Companion which interacts with the user and captures moments

(iii) Built environment which simulates the captured moments

Since companions such as Emobie [10] and Huggable [12] are studied with child users, Paro [11] is studied with adult users and wearable technologies are not limited to a specific user group, the proposed technology will be appropriate for a wide range of users. Also within the study it would be possible to identify the factors that need to be calibrated or customized for different types of user groups.

\subsection{Wearable device which collects body data}

The proposed wearable device is responsible for collecting data from the user's body and share those data with the companion. Data sharing can happen in real time if the companion is available with user, otherwise the wearable device should be capable of storing certain amount of collected data and sharing with the companion whenever it is available. More research can be done at this level in collecting body data (respiratory pattern, heart beat and more factors that reflect the stress level of a person) so that the accuracy of stress level calculations can be improved compared to existing solutions. Also it is needed to ensure that the negative health impacts due to wearing this device is at a minimum, while considering other ergonomics factors.

\subsection{Companion which interacts with the user and captures moments}

The companion which is proposed can be considered as an alternative to a pet for the user, which reacts by analyzing and understanding the stress level in real time or stress level during the time that the user was away from the companion. Machine learning algorithms are needed to develop in order to predict the accurate stress level of the user, with the use of collected body data and feedback received from user regarding calculated stress level in past. Therefore the companion will have its own intelligence to adapt to different types of users from different backgrounds. Some users may prefer to have a standalone companion, while some users may see it as a great idea if the companion can be integrated to commonly used devices like phones. So it is suggested to support both versions, where the companion can be a case to a phone (one possible suggestion - a pet looking phone case looking at the user with blinking eyes, where the phone lies in its stomach) or comes with its own mini screen. Apart from the expressions and reactions done with its eyes and mouth, it will be capable of suggesting different things to user visually with the use of its screen, in order to support to reduce stress level. Also it is suggested to have a sensor kit at the back of its head, so that it will suggest the user to capture the moments when user experiencing calm moments due to environmental factors. Initial idea is to capture inputs like temperature, front view, brightness and audio. Later on with technological advancements it will be possible to improve the capturing by recording wind patterns, which will open up new research areas. 


\subsection{Built environment which simulates the captured moments}

It is suggested to create a built environment inside a small room, which is capable of recreating the captured moments by the companion and also allows the user to control the conditions. So that the user can get relaxed at home by regenerating the environmental conditions that can make him calm. Therefore it is not needed to worry anymore because of not having enough free time to visit the places that user always wants to be. Research needs to be carry out with the use of electronic and electrical instruments to make the conditions appear more natural to the user by applying slight variations to the conditions.

\section{THE WAY AHEAD}

In order to evaluate the usability and functionality of this envisioned research study, it is suggested to use a mixed method approach of empirical evaluation where there are components of both quantitative and qualitative evaluations.

In designing an effective anxiety measuring model, laboratory studies can be conducted with the participation who have been carefully selected. As the participants, it would be suitable to select participants representing different age groups and different anxiety levels like no anxiety, mild anxiety, moderate anxiety, sever anxiety and very sever anxiety. In order to define the anxiety level of a subject, test questionnaires can be used. By setting up a series of activities to perform which may change the stress and anxiety levels while using the prototypes which measures different body measures, it would be able to come up with a practical model to calculate stress and anxiety level of a subject with the use of participants' inputs about how they felt during those activities. Analysis of data gathered from such a laboratory evaluation can be useful in combining the body measures according to observations in order to increase the accuracy.

In designing the companion with its own display or a mobile phone's display, it would be possible to use participatory design techniques asking the users to illustrate the nature of a pet like companion that they like to share their emotions with.

After carefully implementing the initial versions of the companion, wearable device and the controllable environment by studying use cases and by using the experiences and opinions of the psychological specialists, the initial prototypes can be tested against the users with the aim of verifying the positive or negative effects of the system and identifying the improvable areas. Two groups of users can be asked to use the system where one set is provided with the wearable device and the interactive companion whereas the other set is provided only with the wearable device with data storing capabilities, to be used during a certain period. At the end of usage, post-task walkthroughs can be carried out to gather data to perform a qualitative analysis. Since the system is capable of storing data on stress level of the user, that data can be used to perform a quantitative analysis. With the use of both the analysis, pre-defined hypotheses (such as $\mathrm{HO}$ - there is no impact on the stress level of the user by the companion, $\mathrm{H} 1$ - there is an impact on the stress level of the user by the companion) can be proven or disproven and come to conclusions. While it is possible to evaluate the system qualitatively by using query techniques such as interviews, the system itself becomes an evaluator as it has the capability of analyzing quantitatively whether the stress level of the user has reduced after using the system.

The effectiveness of the controlled environment which recreates captured moments through the companion can be measured by comparing the stress levels measured by the system when capturing the moments and when recreating the moments. If it is possible to show that there is a close relationship between above two scenarios, the controlled environment can be recognized as a successful creation.

If prototypes can be exposed to the users and evaluated at early stages of the implementation as proposed, the analyzed outcome of these formative assessments can be used to improve the quality of the work in progress by enhancing the accuracy of stress measuring techniques, interactivity of the companion, quality of the recreated environment, etc.

\section{REFERENCES}

[1] Mental Health Foundation. (2016) Fundamental Facts about Mental Health. Mental Health Foundation, London.

[2] Australian Bureau of Statistics. (2015) National Health Survey: First Results, 2014-15. Australian Bureau of Statistics, Canberra.

[3] World Health Organization. (2014) Preventing Suicide - A global imperative. WHO Press, Geneva.

[4] Nepon J., Lee S. and Bolton J. (Sep. 2010) The Relationship between Anxiety Disorders and Suicide Attempts: Findings from the National Epidemiologic Survey on Alcohol and Related Conditions. Depress Anxiety, vol. 27, no. 9, pp. 791-798.

[5] Baxter A. J., Scott K. M., Vos T. and Whiteford H. A. (July 2012) Global prevalence of anxiety disorders: A systematic review and meta- 
regression. Psychological Medicine, vol. 43, no. 5, pp. 1-14.

[6] Vacca R. (2016) Designing for Interactive Loving and Kindness Meditation on Mobile. $\mathrm{CHI}$ Conference Extended Abstracts on Human Factors in Computing Systems (CHI EA '16), California, USA, May 07 - 12, 2016, pp.17721778. ACM, New York, USA.

[7] Moraveji N., Santoro E., Smith E., Kane A., Crum A. and Susi M. (2017) Using a Wearable Health Tracker to Improve Health and Wellbeing under Stress. 36th Anxiety and Depression Association of America's conference.

[8] Onorati F., Regalia G., Caborni C. and Picard R. (Sep. 2016) Improving convulsive seizure detection by exploiting data from outpatient settings using the Embrace wristband. Poster presented at 12th European Congress on Epileptology.

[9] Klamet J., Matthies D. J. C. and Minge M. (2016) WeaRelaxAble: a wearable system to enhance stress resistance using various kinds of feedback stimuli. 3rd International Workshop on Sensorbased Activity Recognition and Interaction (iWOAR '16), Rostock, Germany, June 23 - 24, 2016. Article No 2. ACM, New York, USA.

[10] Arnold L. (2016) EmobieTM: A Robot Companion for Children with Anxiety. HRI '16 the Eleventh ACM/IEEE International Conference on Human Robot Interaction, Christchurch, New Zealand, March 07 - 10, 2016, 413-414. IEEE Press Piscataway, NJ, USA.

[11] Wada K. and Shibata T. (Oct. 2007) Living with seal robots - Its sociopsychological and physiological influences on the elderly at a care house. IEEE Transactions on Robotics, vol. 23, no. 5, pp. 972-980.

[12] Jeong S., Logan D., Goodwin M., Graca S., O'Connell B., Goodenough H., Anderson L., Stenquist N., Fitzpatrick K., Zisook M., Plummer L., Breazeal C. and Weinstock P. (2015) A Social Robot to Mitigate Stress, Anxiety, and Pain in Hospital Pediatric Care. Tenth Annual ACM/IEEE International Conference on Human-Robot Interaction Extended Abstracts (HRI'15 Extended Abstracts), Oregon, USA, pp.103-104. 